\title{
The ameliorative effect of bioactive phytochemicals (resveratrol, curcumin and sulforaphan) on environmental chemicals evoked inflammation: toxicogenomic data mining approach
}

\author{
Katarina Živančević*, Katarina Baralić, Dragica Jorgovanović, Danijela Đukić-Ćosić \\ Department of Toxicology ,Akademik Danilo Soldatović“, Faculty of Pharmacy, University of Belgrade, \\ Vojvode Stepe 450, 11000 Belgrade, Serbia
}

\section{Introduction}

Inflammation is a well-described adaptive response triggered by endogenous and exogenous antigens and different conditions, such as infection and tissue injury. However, wide varieties of physiological and pathological processes are connected with inflammation. We distinguish acute inflammation, which is manifested by cardinal signs of inflammation, and chronic inflammation that can occur in the absence of some of them (Medzhitov, 2008; Khansari et al., 2009).

The low-grade systemic chronic inflammation can contribute to emergence of serious pathological conditions such as autoimmunity, neurodegeneration, diabetes, cancer and accelerated aging. Although usually inflammation is not a primary cause, it plays an important role in development of these diseases, and treatment focused on suppression of inflammatory reactions and its consequences in many cases can ameliorate these conditions (Kuprash and Nedospasov, 2016).

There is a growing body of evidence demonstrating that environmental chemicals promote different diseases by inducing inflammation (Ghezzi et al., 2018).
Conversely to inflammatory-promotive mode of action, resveratrol, curcumin and sulforaphane are powerful phytochemicals known for their antioxidant and anti-inflammatory properties, thus they can be examined as a potential protective combination in the treatment of inflammation (Chen et al., 2018).

The aim of this in silico study was to analyze the individual and combined therapeutic effects of resveratrol, curcumin and sulforaphane on the regulation of genes associated with the development of inflammation evoked by environmental chemicals using the toxicogenomic data mining approach.

\section{Materials and methods}

The Comparative Toxicogenomic Database (CTD; http://ctd. mdibl.org) and its tools (Batch Query, MyVenn and Set Analyzer) were used to obtain the information about the interactions of investigated phytochemicals with genes/proteins associated with environmental chemical-linked inflammation. Functions of genes were obtained from the GeneCards: the Human Gene Database (https://www.genecards.org), while GeneMania prediction server (https://genemania.org) revealed detailed gene interactions.

* kajaziv93@gmail.com 


\section{Results and discussion}

Inference Score, representing a measure of degree of support for a given association between a disease and a chemical, showed that bisphenol A, cyclophosphamide, trinitrobenzenesulfonic acid, ferrous sulfate, ozone, acrolein, toluene 2,4diisocyanate, asbestos, hydrochloric acid, and pyruvaldehyde have the strongest connectivity with inflammatory processes development among all environmental chemicals listed in the CTD. Additionally, CTD contains list of chemicals marked as therapeutic agents for inflammation. In our study we have focused on protective properties of bioactive phytochemicals (resveratrol, curcumin and sulforaphan). Resveratrol curcumin and sulforaphan interacted with 87, 60 and 31 genes that showed suppressive effect on inflammation, respectively (Batch Query). MyVenn CTD tool revealed that resveratrol, curcumin and sulforaphan interacted with 26 common genes which show inflammatory suppression (ADIPOQ, AGT, AHR, AKT1, BDNF, CCL2, CXCL8, HMOX1, ICAM1, IFNG, IL1B, IL6, JAK2, KYNU, MMP2, NGF, NOS2, PARP1, PPARG, PTGS2, SOD1, TFRC, TGFB1, TIMP1, TLR4, TNF). They participate in 60 different metabolic pathways associated with the modulation of inflammatory response (Set Analyzer), including interleukin signaling pathway (IL-4, IL-6, IL-10, IL13, IL-17), HIF-1 signaling pathway, NF-kappa B signaling pathway, TNF signaling pathway, MAPK signaling pathway, Jak-STAT signaling pathway, PI3K-Akt signaling pathway, signal transduction, adipocytokine signaling pathway that affect a wide range of biological processes, such as cell growth, cell cycle, transcription, cytoskeletal redistribution, cell proliferation, differentiation and apoptosis.

Ghezzi et al. (2018) have shown that the immune system plays an important role in inflammatory response and cancer pathways through toll-like receptor signaling pathway (a particularly important is TLR4). The investigated dietary phytochemicals act anti-inflammatory by modulating immune system through toll-like receptor signaling pathway (AKT1, CXCL8, IL1B, IL6, TLR4, TNF) and cytokine signaling in immune system (AKT1, CCL2, CXCL8, HMOX1, ICAM1, IFNG, IL1B, IL6, JAK2, MMP2, NOS2, PTGS2, TGFB1, TIMP1, TNF). GeneMania server revealed that most of common genes were in co-expression - simultaneous expression of genes $(68.38 \%)$, co-localisation genes found in the same location $(17.22 \%)$ and physical interaction - protein-protein interactions $(8.88 \%)$.

\section{Conclusion}

These results confirm both individual and combined anti-inflammatory effect of investigated phytochemicals that could be considered for further in vitro and in vivo investigation in order to clarify the mechanisms of their beneficial effects, especially the different acting manner of their common 26 genes.

\section{References}

Chen, C.Y., Kao, C.L., Liu, C.M., 2018. The cancer prevention, anti-inflammatory and anti-oxidation of bioactive phytochemicals targeting the TLR4 signaling pathway. Int. J. Mol. Sci. 19(9). Available at: https://doi.org/10.3390/ijms19092729.

Ghezzi, P., Floridi, L., Boraschi, D., Cuadrado, A., Manda, G., Levic, S., D’Acquisto, F., Hamilton, A., Athersuch, T.J.., Selley, L., 2018. Oxidative stress and inflammation induced by environmental and psychological stressors: A biomarker perspective. Antioxid. Redox Signal. 28(9), 852-872. Available at: https://doi.org/10.1089/ars.2017.7147.

Khansari, N., Shakiba, Y., Mahmoudi, M., 2009. Chronic inflammation and oxidative stress as a major cause of age- related diseases and cancer. Recent. Pat. Inflamm. Allergy Drug Discov. 3(1), 73-80. Available at: https://doi.org/10.2174/187221309787158371.

Kuprash, D.V., Nedospasov, S.A., 2016. Molecular and cellular mechanisms of inflammation. Biochem (Mosc). 81(11), 1237-1239. Available at: https://doi.org/10.1134/S0006297916110018.

Medzhitov, R., 2008. Origin and physiological roles of inflammation. Nature. 454(7203), 428-435. Available at: https://doi.org/10.1038/nature07201. 